\title{
Review: music as a single session intervention reduces anxiety and respiratory rate in patients admitted to hospital
}

\author{
Evans D. The effectiveness of music as an intervention for hospital patients: a systematic review.J Adv Nurs 2002 \\ Jan;37:8-18.
QUESTION: In adult patients admitted to hospital, does listening to music reduce the perception and physiological consequences of pain and anxiety, minimise the effect of unpleasant procedures and situations, and increase satisfaction with care?

\section{Data sources}

Studies were identified by searching Medline, CINAHL, Current Contents, EMBASE/Excerpta Medica, Cochrane Library, Dissertation Abstracts, PsycLit, Proceeds, HealthStar, Austhealth, and Expanded Academic Index; handsearching 4 journals that focus on music and health care; and reviewing the reference lists of retrieved articles.

stated

For correspondence:

Dr D Evans, Joanna

Briggs Institute, Royal

Adelaide Hospital,

Adelaide, South

Australia.

d.evans@adelaide.edu.au

\section{Study selection}

Studies were selected if they were randomised controlled trials evaluating the effectiveness of recorded music compared with a control intervention in adult patients in a hospital setting. At least 1 of the following outcomes

\section{COMMENTARY}

This review by Evans of single session music interventions for patients used a comprehensive and rigourous search method and included only randomised controlled trials. The precise scope is slightly ambiguous as quality criteria used to select trials were unstated, quality of included studies was not described and $\geqslant 1$ of the studies included in the meta-analysis delivered 2 sessions of music intervention.

Music interventions have been used among diverse patient populations. ${ }^{1}$ Studies included in the review by Evans predominately involved patients in critical care or surgical units and showed evidence of reduction in anxiety. The review showed no benefits for patients having procedures. Application of these findings to other patient populations in different clinical environments and with different diagnoses and illness experiences needs further careful consideration by clinicians.

Clinicians may want to consider the type of patients under their care as well as the aim of their intervention. For example, among cardiac and ventilated patients in critical care, music interventions have predominately been used as an anxiety/stress reduction intervention. ${ }^{2}$ However, among comatose patients or those with neurological deficits, the aim of a music intervention may be for stimulation.

Cultural considerations of music preferences are important, as previous research has identified differences among ethnic groups. ${ }^{3}$ This concurs with current recommendations to allow patients to select music in order to achieve positive outcomes. ${ }^{2}$ Furthermore, clinicians may want to encourage patients or family members to bring a personal tape or CD player with their own music to the hospital. ${ }^{2}$ The effect of such interventions has not been tested in this review, although its findings broadly support the potentially positive role that music may play.

Ruth Taylor-Piliae, RN, CNS, MN Doctoral Student, School of Nursing University of California, San Francisco San Francisco, California, USA

1 Snyder M, Chlan L. Music therapy. Annu Rev Nurs Res 1999;17:3-25.

2 Chlan L, Tracy MF. Music therapy in critical care: indications and guidelines for intervention. Crit Care Nurse 1999;19;35-41.

3 Good M, Picot BL, Salem SG, et al. Cultural differences in music chosen for pain relief. J Holist Nurs 2000;18:245-60.

had to be reported: anxiety, pain, satisfaction, vital signs, analgesic use, sedation use, tolerance, mood, or length of stay. Studies with poor methodologies were excluded.

\section{Data extraction}

Data were extracted in duplicate on patient characteristics, type of music, and outcome measures.

\section{Main results}

19 studies met the selection criteria. 12 studies evaluated the effect of music on anxiety, 6 of which involved patients admitted to hospital. Combining the results from these 6 studies showed that music reduced anxiety (standardised mean difference [SMD] $-0.71,95 \%$ CI -0.97 to -0.46 ). The other 6 trials evaluated music in patients having a procedure, 2 of which could be combined. Combining these 2 studies showed that music did not reduce anxiety (SMD 0.06, CI -0.33 to 0.44). Based on the findings of 2 trials that could not be combined, music did not reduce anxiety in patients having invasive or unpleasant procedures.

5 studies evaluated the effect of music on respiratory rate. Combining the results of 3 of these studies showed a reduction in respiratory rate with music in patients admitted to hospital (weighted mean difference [WMD] -2.42 , CI -3.95 to -0.88 ). 1 study found no difference between treatment and control groups for those having a procedure.

11 studies evaluated the effect of music on heart rate, 8 evaluated the effect of music on systolic blood pressure, and 2 evaluated the effect of music on the perception of pain. No differences existed between the treatment groups for either patients admitted to hospital or those having a procedure.

2 studies evaluated the effect of music on sedation and showed that music may decrease the need for or the amount of sedatives. 1 study found no difference in patient tolerance of having a procedure between those in the music and control groups. 1 study found that music increased patient satisfaction, but another found no difference in satisfaction between the treatment groups. 2 studies found that music led to an improvement in patient mood. 1 study found no difference between treatment groups in the duration of hospital stay.

\section{Conclusion}

Music as a single session intervention reduces anxiety and respiratory rate in patients admitted to hospital. 